\title{
señalización para indicar los pasos de cebra para pentones
}

MANUEL MATEOS, Dr. Ingeniero de caminos

\section{sinopsis}

En el presente artículo se resumen las experiencias sacadas por el Road Research Laboratory, sobre pasos para peatones no regulados por semáforos. Internacionalmente se señalan sobre la calzada mediante pasos cebra, formados por una serie de franjas longitudinales con colores amarillo y negro alternados.

Se recomienda también un tipo de señalización, que permita al conductor apreciar intuitivamente la proximidad de estos pasos y conocer su situación exacta sobre la calzada en cualquier circunstancia.

La solución propuesta consiste en representar el paso cebra con tamaño reducido, en señales verticales visibles durante la noche gracias a un material reflectante.

En los dibujos y fotografías que acompañan al artículo se pueden apreciar la forma de estas señales y los esquemas para su colocación.

Los pasos de cebra hechos para resaltar los cruces para peatones son fruto de la investigación vial in glesa. En el año 1946, el Laboratorio de Investigaciones de Carreteras (Road Research Laboratory) inició una serie de estudios sobre cruces para peatones a instancia del Ministerio de Transportes. Estos estudios tenían como objeto principal el encontrar unas marcas que hicieran los pasos para peatones más visibles, tanto para los conductores como para los peatones.

Se analizaron distintas clases de marcas, entre ellas los clavos usados en aquella época, y se obtuvieron las conclusiones de que las marcas más efectivas eran las bandas hechas longitudinalmente, o sea, paralelas al eje y bordillos de la calzada. También se halló que los colores más efectivos eran blanco y negro alternados y la anchura de bandas más recomendable los $60 \mathrm{~cm}$. Se comprobó que los pasos de cebra seguían siendo unas marcas poco visibles durante la noche o con pavimento mojado. Para paliar esto se recomendó la iluminación nocturna de los pasos para peatones, así como el empleo, en la franja negra, de materiales con textura tal que actuaran como reflectores difusores de la luz.

Como resultado de estos estudios se establecieron en 1951 nuevas regulaciones para los pasos de peatones no controlados con semáforos. Se recomendó el uso de franjas tipo cebra, así como que en estos pasos los vehículos dieran preferencia a los peatones. También se recomendó que el número de pasos no controlados se redujera en dos tercios, y que fuera ilegal aparcar vehículos a menos de $25 \mathrm{~m}$ de los pasos para peatones en calles con velocidades máximas superiores a $40 \mathrm{~km} / \mathrm{h}$. En trabajos posteriores se halló la conveniencia de proveer a estos pasos de luces intermitentes para llamar la atención sobre su existencia durante la noche.

Las regulaciones de 1951 se establecieron después de una campaña efectiva de educación e información de los usuarios.

Los pasos de cebra se copiaron en seguida en multitud de países, siendo hoy internacional tal manera de distinguir los pasos para peatones no controlados por semáforos.

En España se ven pasos de cebra en muchísimas ciudades, aunque a veces la adopción o copia de tales señales no se hace teniendo en cuenta todas las recomendaciones del Road Research Laboratory, sobre todo en lo que se refiere a la campaña de educación, necesidad de que no

Señal que se recomienda para indicar los pasos de peatones no controlados por semáforos.

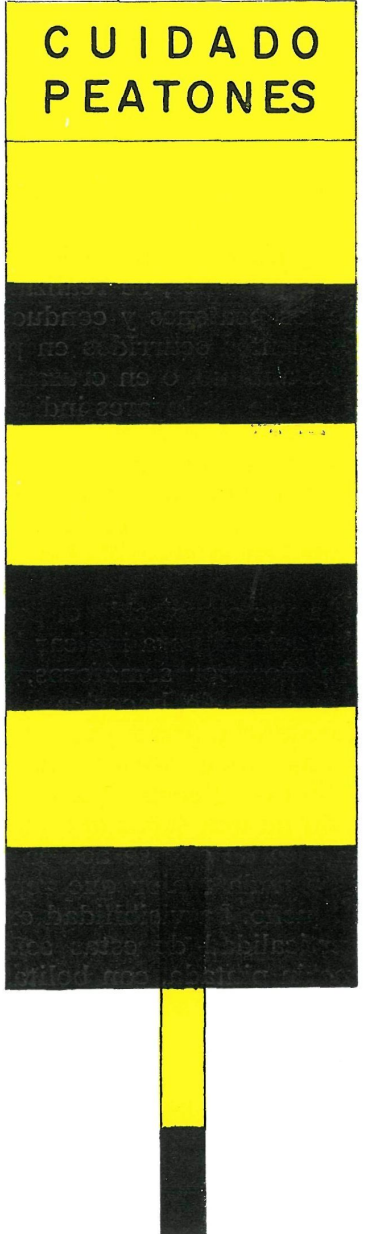



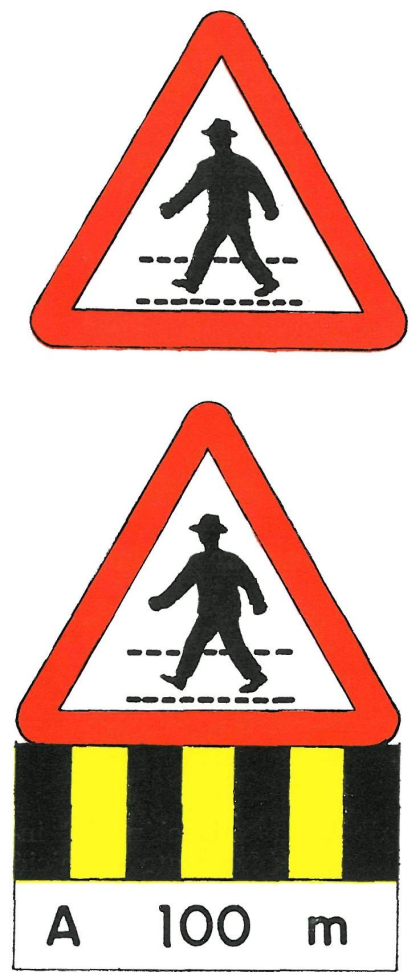

Señal existente y señal que se propone para indicar la proximidad de un paso para peatones.

aparquen coches en sus inmediaciones, anchura y longitud de las franjas, y limitación de su proliferación.

Después de la adopción en 1951 de los pasos de cebra para peatones, se realizaron estudios sobre la conducta de los peatones y conductores, así como del número de accidentes ocurridos en pasos con cebra y en pasos del tipo antiguo, o en cruzamientos hechos por los peatones fuera de los lugares indicados como pasos para peatones.

Estos estudios corroboraron las conclusiones obtenidas en el Laboratorio y demostraron que también se obtenía una pequeña reducción en el número de accidentes, así como en la severidad de éstos.

Una vez establecido el paso en cebra como distintivo internacional para indicar los pasos para peatones, no controlados por semáforos, queda todavía por resolver el problema de hacerlos más visibles bajo cualquier circunstancia. Para esto proponemos la adopción de las señales que presentamos en las fotografías y dibujos adjuntos. Nuestra recomendación se basa en la introducción de una señal que asocie fácilmente en el conductor la idea de que se acerca a un paso para peatones. Para esto nada mejor que reproducir el dibujo de cebra en pequeño. La visibilidad en todo tiempo está dada por la verticalidad de estas señales; y rociando la superficie, recién pintada, con bolitas microscópicas, se hace reflectante y muy visible durante la noche.

Esquema de la disposición de las señales recomendadas. bibliog rofica

nistry of Transport: "The Pedestrian Crossing Regulation". Statutory oad Research Laboratory: "Research on Road Safety». H. M. Stationery Office. Londres, 1963.

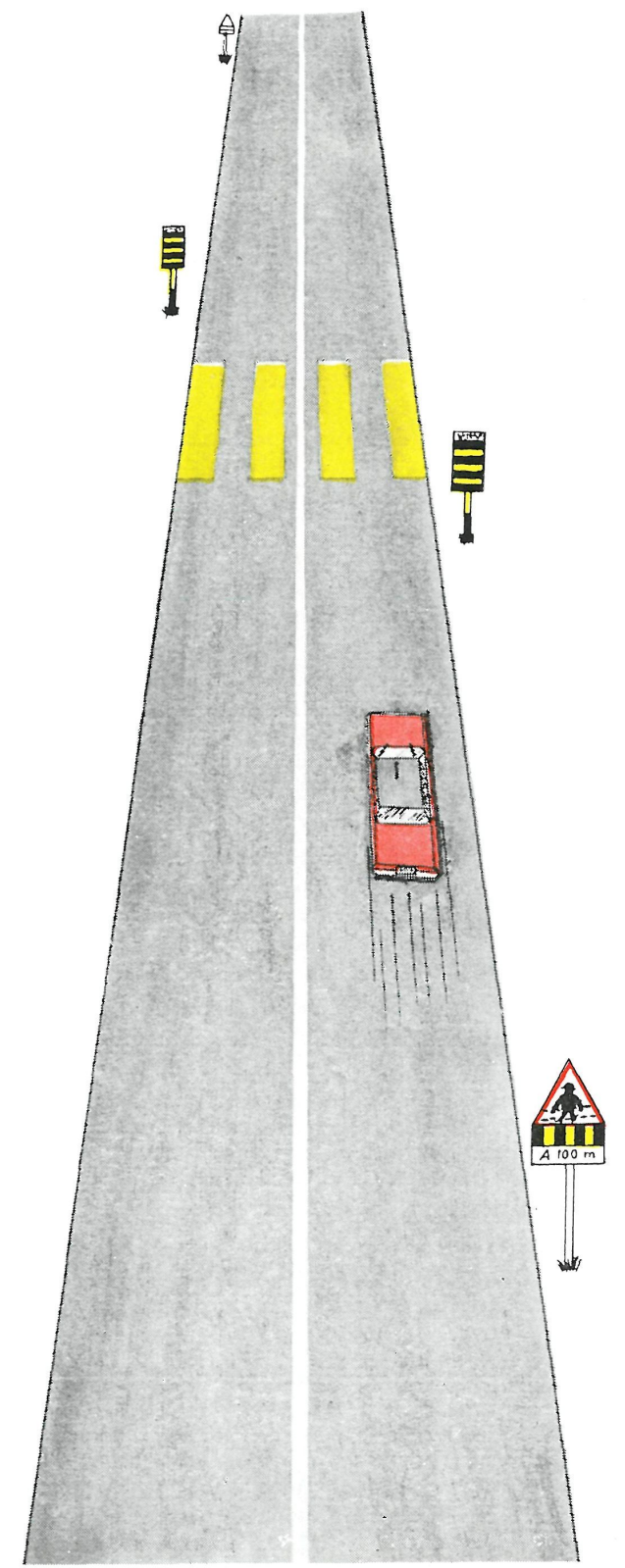




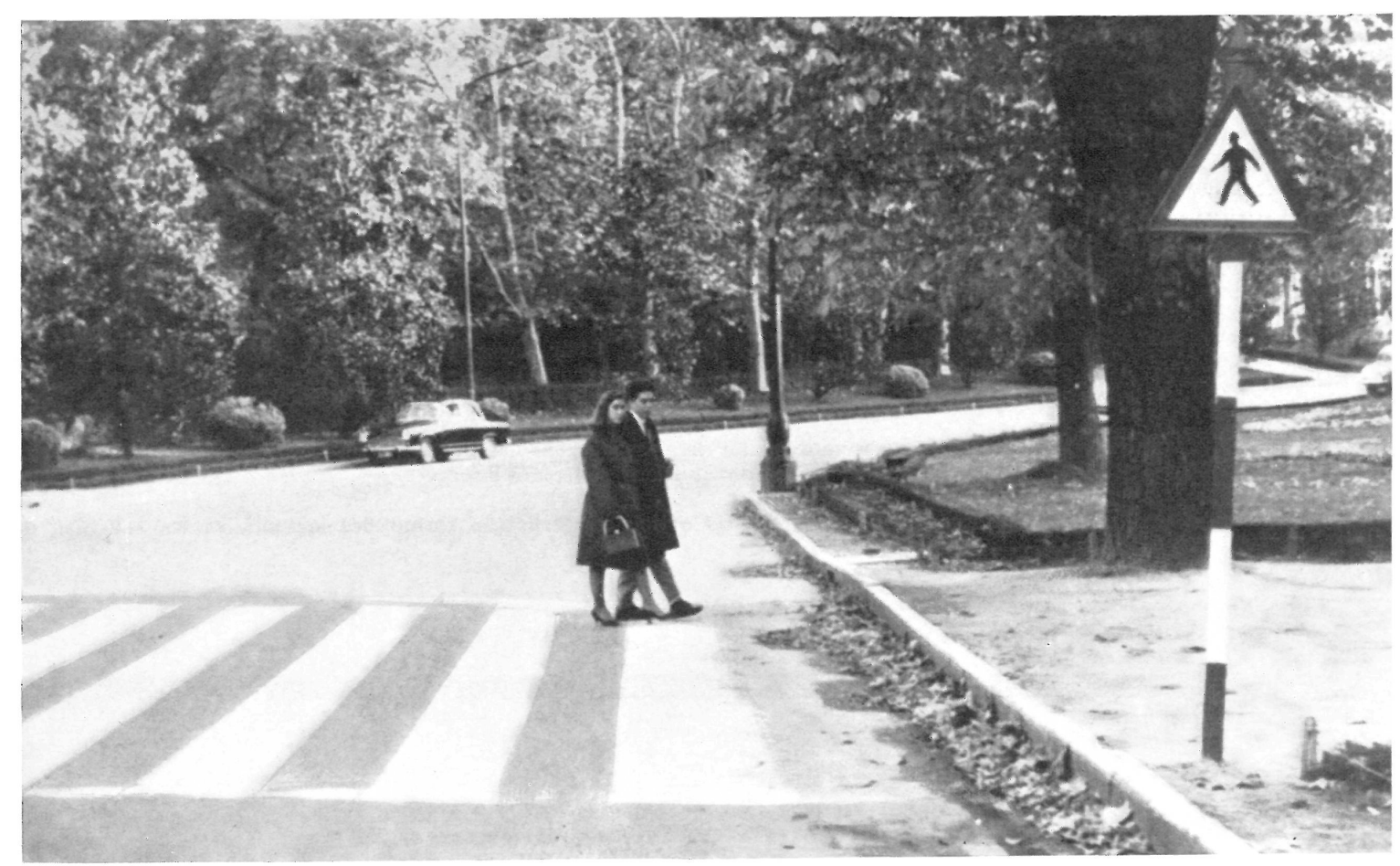

Paso de cebra para peatones.

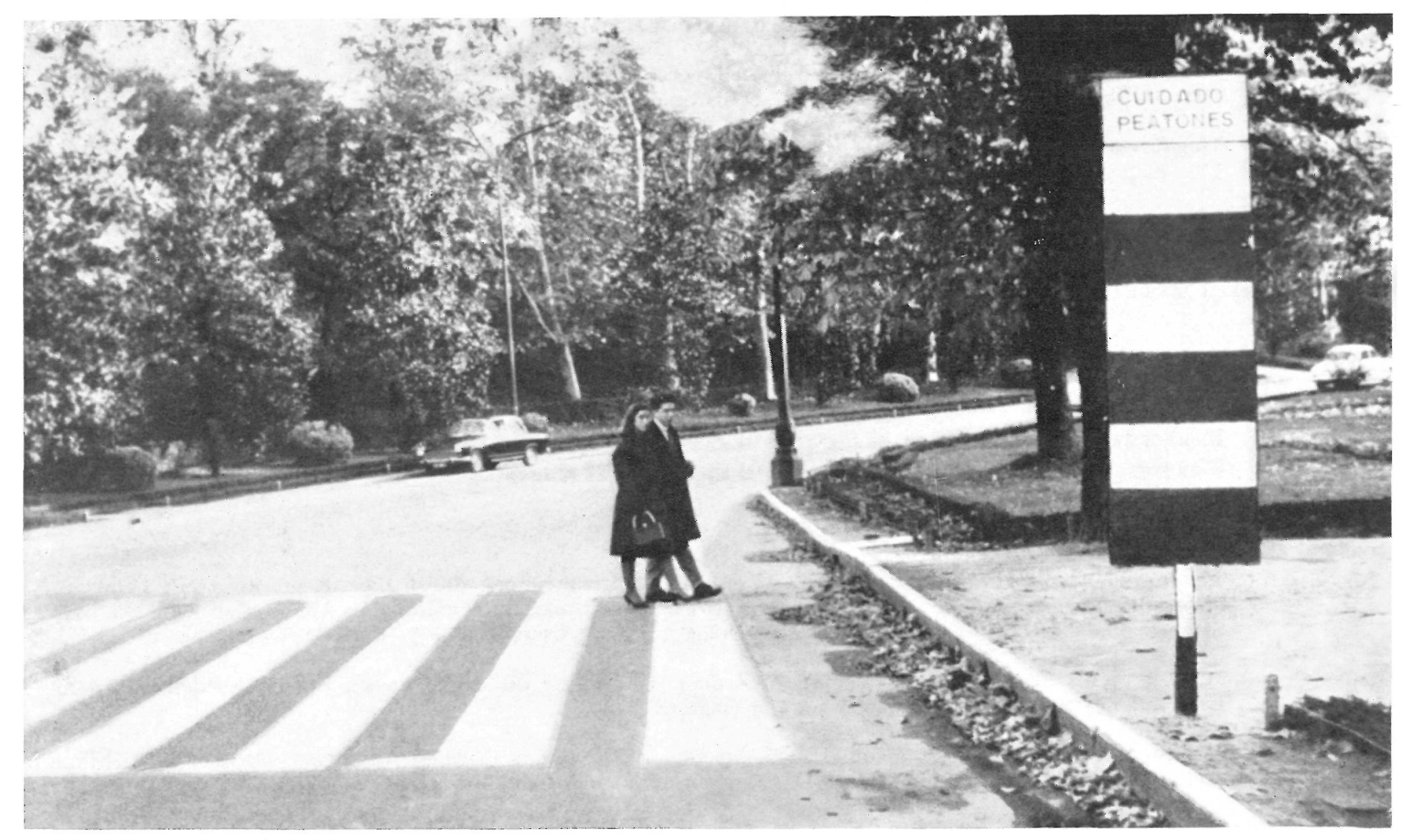

Paso con la señal que recomendamos. 


\section{Signaliscatriom des passages zélbrés pour piétoms}

Manuel Mateos, Dr. ingénieur des Ponts et Chaussées

Cet article résume les expériences tirées par le Road Research Laboratory sur les passages pour piétons non réglés par des feux de circulation. Internationalement ces passages sont signalés sur la chaussée par des zones zébrées, formées par une série de raies longitudinales jaunes et noires alternées.

Un type de signalisation est également recommandé pour permettre au conducteur d'apprécier intuitivement la proximité de ces passages et d'en connaître, en n'importe quelle circonstance, la situation exacte sur la chaussée.

La solution proposée consiste à représenter le passage zébré, en dimensions réduites, à l'aide de signaux verticaux visibles pendant la nuit grâce à une matière réfléchissante.

Les dessins et les photographies illustrant cet article montrent la forme des signaux et les schémas de leur mise en place.

\section{Sigmalling of zebru pedestreicu crossings}

Manuel Mateos, Dr. civil engineer

This article summarises the experience collected by the Road Research Laboratory on pedestrian crossings that are not regulated by a traffic light. These crossings are marked, by international convention, by a number of longitudinal stripes, alternatively yellow and black.

It is also recommended that additional signalling be provided, enabling the driver to know that he is approaching such a crossing, and appreciate its precise location.

The proposed method is to show a reduced version of the zebra crossing on vertical signposts, which can be seen at night, by painting them with a reflecting material.

The drawings and photographs in the article show such signs, and how they can be located along the roadside.

\section{Zeichem unn die durch zelbracurtige Streifem \\ Fussgg cingeriberwege zu zeichmem}

Manuel Mateos, Dr. Bauingenieur

Im vorliegenden Artikel fässt man die genommenen Erfahrungen durch das «Road Research Laboratory» über Fussgängerüberwege ohne Verkehrssignale zusammen. Durch internationalen Abkommen zeichnet man auf der Strasse eine längliche zebraartige Streifenreihe in abwechselnder Gelb-und Schwarzfarbe.

Man empfehlt auch einen Zeichentyp, der dem Autofahrer die Nähe diesen Streifen sehen und seine genaue Lage auf der Strasse in jeder Zeit kennen erlaubt.

Die vorgeschlagene Lösung gründet sich auf den zebraartigen Streifen in kleiner Grösse, in sichtbaren senkrechten aus fluoreszierenden Material Zeichen, um während der Nacht zu sehen.

In den Zeichnungen und Bildern, die in diesem Artikel stehen, kann man die Form dieser Zeichen und der Schemas für ihre Aufstellung beachten. 